\title{
On a Duality Between Time And SPACE CONES
}

\author{
Waleed Al-Qallaf, Kyriakos Papadopoulos \\ Department of Mathematics, Kuwait University, PO Box 5969, Safat 13060, Kuwait \\ E-mail: kyriakos@sci.kuniv.edu.kw
}

\begin{abstract}
We give an exact mathematical construction of a spacelike order $<$, which is dual to the standard chronological order $\ll$ in the $n$-dimensional Minkowski space $M^{n}$, a construction that can be extended to any curved spacetime, and we discuss its geometrical as well as its topological implications.
\end{abstract}

\section{Introduction.}

Many important theorems, within the frame of general relativity, refer to spacelike, such as singularity theorems which require the existence of a smooth spacelike Cauchy surface $\Sigma$ (see [10]), schematic conformal diagrams depicting causal independence (see for example [6]), etc. In all cases, spacelike is synonymous to acausal, where there is no timelike relation or information traveling with the speed of light. In this article we show that the structure of the null-cone is induced, in a topological sense, by a spacelike order which creates a spacelike orientation in an analogous way to the timelike orientation.

\section{Definitions and notation.}

Let $M^{n}$ be the $n$-dimensional Minkowski space. Let $Q$ be the characteristic quadratic form on $M^{n}$, defined by $Q(x)=\left\{x_{0}^{2}+x_{1}^{2}+x_{2}^{2}+\cdots+x_{n-2}^{2}-x_{n-1}^{2}: x=\left(x_{0}, x_{1}, x_{2}, \cdots, x_{n-1}\right) \in M^{n}\right\}$.

For an event $x \in M^{n}$, we consider the following sets: 
1. $C^{T}(x)=\{y: y=x$ or $Q(y-x)<0\}$, the time cone of $x$,

2. $C^{L}(x)=\{y: Q(y-x)=0\}$, the light cone of $x$,

3. $C^{S}(x)=\{y: y=x$ or $Q(y-x)>0\}$, the space cone of $x$ and

4. $C^{N}(x)=C^{S}(x) \cup C^{L}(x)$ the null cone of $x$.

We also consider the half-planes:

1. $P_{m}^{+}(x)=\{y: g(m, y-x) \geq 0$ and $y \neq x\}$ and

2. $P_{m}^{-}(x)=\{y: g(m, y-x) \leq 0$ and $y \neq x\}$

in $M^{n}$, for any plane $P_{m}(x)$, where $m \in M^{n}, m \neq 0$ is the normal to $P_{m}(x), P_{m}(x)=$ $\{y: g(m, y-x)=0\}$ and where $g$ denotes the spacetime metric.

For abbreviation, we will write $P_{m}^{+}(x):=P^{+}(x), P_{m}^{-}(x):=P^{-}(x)$ and $P_{m}(x):=P(x)$.

We observe that $P_{+}(x) \cup P_{-}(x)=\{x\}^{c}$. So, for an event $y \in M, y \in P_{+}(x) \cup P_{-}(x)=\{x\}^{c}$ if and only if $y \neq x$.

Last, but not least, we define the following subspaces:

1. $C_{+}^{L}(x)=P_{+}(x) \cap C^{L}(x)$;

2. $C_{-}^{L}(x)=P_{-}(x) \cap C^{L}(x)$;

3. $C_{+}^{S}(x)=P_{+}(x) \cap C^{S}(x)$;

4. $C_{-}^{S}(x)=P_{-}(x) \cap C^{S}(x)$;

5. $C^{L S}(x)=C^{L}(x) \cup C^{S}(x)$;

6. $C_{+}^{L S}(x)=P_{+}(x) \cap C^{L S}(x)$;

7. $C_{-}^{L S}(x)=P_{-}(x) \cap C^{L S}(x)$.

It is standard (see [1]) to consider two partial orders, the chronological order $\ll$, which is irreflexive, and the causal order $\prec$, which is reflexive, defined not only in $M^{n}$ but in general in any spacetime, as follows: 
1. $x \ll y$ iff $y \in C_{+}^{T}(x)$ and

2. $x \prec y$ iff $y \in C_{+}^{T}(x) \cup C_{+}^{L}(x)$

In addition, the reflexive relation horismos $\rightarrow$ is defined as $x \rightarrow y$ iff $x \prec y$ but not $x \ll$ $y$.

\section{The weak interval topology.}

We will consider a topology that we call the weak interval topology, which is constructed in an analogous way to the interval topology in [2], but which does not apply only to lattices. In fact, when restricted to the 2-dimensional Minkowski space $M^{2}$, under the causal order $\prec$, the weak interval topology will coincide with the interval topology, but in general it will not be restricted to lattices. For its construction, we need a relation $R$ defined on a set $X$. We then consider the sets $I^{+}(x)=\{y \in X: x R y\}$ and $I^{-}(x)=\{y \in X: y R x\}$, as well as the collections $\mathcal{S}^{+}=\left\{X \backslash I^{-}(x): x \in X\right\}$ and $\mathcal{S}^{-}=\left\{X \backslash I^{+}(x): x \in X\right\}$. A basic-open set $U$ in the weak interval topology $T^{i n}$ is defined as $U=A \cap B$, where $A \in \mathcal{S}^{+}$and $B \in \mathcal{S}^{-}$; in other words, $\mathcal{S}^{+} \cup \mathcal{S}^{-}$forms a subbase for $T^{\text {in }}$.

The topology $T^{\text {in }}$ with respect to the relation $\rightarrow$ was studied in [8] and also in [4] and with respect to the order $\ll$ in [9], where there was conjectured a possibility to create a duality between timelike and spacelike, based on a spacelike order $<$ dual to chronology $\ll$. In this paper we give the exact mathematical construction of $<$ as well as of the topology $T_{<}^{i n}$, for $n$-dimensional Minkowski space $M^{n}$ and, as we will see in our last section, these results can be extended to any $n$-dimensional curved spacetime without any additional condition.

\section{The order on the space-cone and its induced topol- ogy.}

We define a partial spacelike order $<$ dual to the chronological order $\ll$. This order is obviously not causal, but brings an interesting duality between the time cone $C^{T}(x)$ and the space cone $C^{S}(x)$, of an event $x$. Through $<$, the "cone" $C^{S}(x)$ exhibits similar properties to $C^{T}$. Since "chronological" comes from the word "chronos", which means time, we name $<$ "chorological", as it refers to "choros", space. 
Definition 4.1. For events $x, y \in M^{n}, x<y$ iff $y \in C_{+}^{S}(x)$.

It follows that $x<y$ iff $x \in C_{-}^{S}(y)$.

Lemma 4.1. $C_{+}^{L S}(x) \cup C_{-}^{L S}(x)=C^{L S}(x)-\{x\}$.

Proof. $C_{+}^{L S}(x) \cup C_{-}^{L S}(x)=C^{L S} \cap\left(P_{+}(x) \cup P_{-}(x)\right)=C^{L S}(x) \cap\{x\}^{c}=C^{L S}(x)-\{x\}$.

Proposition 4.1. $\left[C_{+}^{L S}(x)\right]^{c} \cap\left[C_{-}^{L S}(x)\right]^{c}=C^{T}(x)$

Proof. $\left[C_{+}^{L S}(x)\right]^{c} \cap\left[C_{-}^{L S}(x)\right]^{c}$

$=\left[C_{+}^{L S}(x) \cup C_{-}^{L S}(x)\right]^{c}$

$=\left[C^{L S}(x)-\{x\}^{c}\right]^{c}$

$=\left[C^{L S}(x)\right]^{c} \cup\{x\}$

$=\left[C^{T}(x)-\{x\}\right] \cup\{x\}$

$=C^{T}(x)$

Remark 4.1. Interestingly enough, we remark that the plane $P(x)$ need not be "perpendicular" to space, in the sense that it will cut the time-cone $C^{T}(x)$ in two equal slices.

Proposition 4.2. The weak interval topology $T_{<}^{i n}$, generated by the space-like order $<$, has as basic-open sets the null-cones $C^{N}(x)$.

Proof. The proof follows from Proposition 4.1, by observing that $\left[C_{+}^{S}(x)\right]^{c} \in \mathcal{S}^{-}$and $\left[C_{-}^{S}(x)\right]^{c} \in$ $\mathcal{S}^{+}$, where $I^{+}(x)$ and $I^{-}(x)$ are defined with respect to $R=<$ (see paragraph 3 ).

Remark 4.2. If we define a relation $R$, such that $x R y$, iff $y \in\left[C_{+}^{S}(x) \cup C_{+}^{L}(x)\right] \backslash\{x\}$, then applying Proposition 4.2 we observe that $R$ will induce a topology $T_{R}^{i n}$ which has as basic-open sets the time-cones $C^{T}(x)$. We only need to observe that $\left[C_{+}^{L S}(x)\right]^{c} \in \mathcal{S}^{-}$and $\left[C_{-}^{L S}(x)\right]^{c} \in \mathcal{S}^{+}$, where $I^{+}(x)$ and $I^{-}(x)$ are defined with respect to $R$.

For the last result of our discussion, we will need to use Reed's definition of intersection topology (see [5]:

Definition 4.2. If $T_{1}$ and $T_{2}$ are two topologies on a set $X$, then the intersection topology $T^{\text {int }}$ with respect to $T_{1}$ and $T_{2}$, is the topology on $X$ such that the set $\left\{U_{1} \cap U_{2}: U_{1} \in T_{1}, U_{2} \in\right.$ $T_{2}$ \} forms a base for $(X, T)$. 
The topology $Z^{T}$ is defined to be the intersection topology, according to Reed's definition, of the topologies $T_{R}^{i n}$ and $\mathbb{R}^{n}$, in $M^{n}$. This topology, in $M$, coincides with one of the three topologies that were suggested by Zeeman in [3], as alternatives to his Fine topology. Its characteristic is that its open sets are space-cones bounded by Euclidean-open balls, in $M^{n}$, and its general relativistic analogue is actually the Path topology of Hawking-King-McCarthy (see $[7])$.

The topology $Z^{L T}$ is defined to be the intersection topology, according to Reed's definition, of the topologies $T_{<}^{i n}$ and $\mathbb{R}^{n}$, in $M^{n}$. This topology fully incorporates the causal structure of $M^{n}$.

\section{Discussion.}

The results of section 4 generalise to any curved spacetime, in the frame of general relativity, for the following reasons. In a relativistic spacetime manifold, wherever there is spacetime there are events and for every event there is a null-cone. Since our construction of $T_{<}^{i n}$ is topological, depending exclusively on the interior (time cone), boundary (light cone) and exterior (space cone) of an event $x$, independently of the geometry of the space-time, and since the causality is defined by the order $\ll$ (with dual acausal order $<$ ), we have that the induced weak interval topology $T_{<}^{i n}$ in a curved spacetime will be defined following exactly the same process as in $M^{n}$ (section 3). In conclusion, the order-theoretic properties of < that we examined in $M^{n}$ hold for any $n$-dimensional relativistic spacetime manifold, as well as the topological properties of $T_{<}^{i n}, Z^{T}$ and $Z^{L T}$.

\section{References}

[1] R. Penrose, Techniques of Differential Topology in Relativity, CBMS-NSF Regional Conference Series in Applied Mathematics, 1972.

[2] Gierz, Gerhard and Hofmann, Karl Heinrich and Keimel, Klaus and Lawson, Jimmie D. and Mislove, Michael W. and Scott, Dana S. A compendium of continuous lattices. Springer-Verlag, 1980.

[3] E.C. Zeeman, The Topology of Minkowski Space, Topology, Vol. 6, 161-170(1967). 
[4] Ignatios Antoniadis, Spiros Cotsakis and Kyriakos Papadopoulos, The Causal Order on the Ambient Boundary, Mod. Phys. Lett. A, Vol 31, Issue 20, 2016.

[5] G.M. Reed, The intersection topology w.r.t. the real line and the countable ordinals (Trans. Am. Math. Society, Vol. 297, No 2, 1986, pp 509-520).

[6] Roger Penrose, The Road to Reality: a complete guide to the laws of the universe Vintage Books, 2007 edition.

[7] Hawking, S. W. and King, A. R. and McCarthy, P. J. (1976) A new topology for curved spacetime which incorporates the causal, differential, and conformal structures. Journal of Mathematical Physics, 17 (2). pp. 174-181.

[8] Kyriakos B. Papadopoulos, Santanu Acharjee and Basil K. Papadopoulos, The Order On the Light Cone and Its Induced Topology, International Journal of Geometric Methods in Modern Physics 15, 1850069 (2018).

[9] Kyriakos B. Papadopoulos and Basil K. Papadopoulos, On Two Topologies that were suggested by Zeeman, accepted, to appear in Mathematical Methods in the Applied Sciences (arxiv: 1706.07488).

[10] S.W. Hawking and G.F.R. Ellis, The Large Scale Structure of Space-Time, Cambridge University Press, 1973. 